\title{
Clinical Diagnostic Tests Versus MRI Diagnosis of ACL Tears
}

\author{
Megan P. Brady and Windee Weiss
}

\begin{abstract}
Clinical Scenario: The anterior cruciate ligament (ACL) injury is a common knee injury within varying athletic levels. Clinical diagnostic tests and magnetic resonance imaging (MRI) are two methods of evaluating ACL injuries. Clinical Question: Are clinical diagnostic tests as accurate as MRI when diagnosing ACL tears? Summary of Key Findings: Three cross-sectional design studies were included. One study found that clinical diagnostic tests were superior to MRI when diagnosing an ACL tear. Another study found that clinical diagnostic tests were equal to MRI when measuring sensitivity, but scored higher in specificity, positive predictive value, negative predictive value, and diagnostic accuracy. The last study found that clinical diagnostic tests scored higher than MRI on specificity and positive predictive value, were equal when measuring accuracy, and scored lower when measuring sensitivity and negative predictive value. Clinical Bottom Line: The evidence supports the use of clinical diagnostic tests when diagnosing an ACL tear. Strength of Recommendation: Level 2-3.
\end{abstract}

Keywords: anterior cruciate ligament, evaluation, knee joint, clinical evaluation, magnetic resonance imaging

\section{Clinical Scenario}

A common injury among elite, recreational, and youth athletes is the anterior cruciate ligament (ACL) tear. ${ }^{1}$ Approximately 200,000 ACL injuries occur every year. ${ }^{2}$ The gold standard in ACL injury evaluation is diagnostic arthroscopy ${ }^{3,4}$; however, the diagnostic accuracy of clinical diagnostic tests and magnetic resonance imaging (MRI) is debatable. ${ }^{1,3-6}$ Because of the frequency of knee injury, ${ }^{3}$ MRI is a common diagnostic tool used for imaging and diagnosis. ${ }^{5}$ ACL tears are also diagnosed using clinical diagnostic tests. ${ }^{1,3-6}$ Patient outcomes are dependent on a clinician's ability to detect an ACL tear, as the length of time required to obtain an MRI and results can be extensive. If an ACL tear is initially diagnosed accurately with clinical diagnostic tests, then the patient could be removed from athletics or work activities to prevent further injury to the knee and could be referred to an orthopedic surgeon for further evaluation. If clinical diagnostic tests are as accurate as MRI when diagnosing an ACL tear, both time and money could be saved for the patient. The patient could be referred to an orthopedic surgeon sooner, and surgical reconstruction could be completed. By saving time and not waiting for an MRI and results, the patient could return to athletics or work activities sooner. If the ACL is diagnosed as intact via clinical diagnostic tests, the patient could save money by avoiding an MRI. Clinical diagnostic tests with a high specificity could allow patients to return to athletics or work sooner, saving time and money by avoiding an MRI test and results. These factors combined could improve patient outcomes.

\section{Focused Clinical Question}

Are clinical diagnostic tests as accurate as MRI when diagnosing ACL tears?

Brady is with Drake University, Des Moines, IA. Weiss is with the University of Northern Iowa, Cedar Falls, IA. Brady (megan.brady@drake.edu) is corresponding author.

\section{Summary of Search, "Best Evidence" Appraised, and Key Findings}

- One study found that clinical diagnostic tests were superior to MRI when diagnosing an ACL tear. ${ }^{6}$

- One study found that, when conducted proficiently, clinical diagnostic tests were equal to MRI when measuring sensitivity, but scored higher in specificity, positive predictive value (PPV), negative predictive value (NPV), and diagnostic accuracy. ${ }^{5}$

- One author determined that when diagnosing ACL tears, clinical diagnostic tests scored higher than MRI on specificity and PPV, were equal when measuring accuracy, and scored lower when measuring sensitivity and NPV. ${ }^{4}$

\section{Clinical Bottom Line}

The evidence supports the use of clinical diagnostic tests when diagnosing an ACL tear. Clinical diagnostic tests are equally or more diagnostically accurate as an MRI in the diagnosis of an ACL tear.

\section{Strength of Recommendation}

Grade $\mathrm{C}$ evidence supports that clinical diagnostic tests are equally or more diagnostically accurate than MRI in the diagnosis of ACL tears. Limited sample sizes are available, and further investigation is needed. Therefore, these findings should not be considered definitive.

\section{Search Strategy}

\section{Terms Used to Guide Search Strategy}

- Patient/Client group: individuals with an ACL injury

- Assessment: clinical diagnostic tests 
- $\underline{\text { Comparison: MRI }}$

- Outcome: ACL tear diagnosis

\section{Sources of Evidence Searched (Databases)}

- Medline/PubMed

- ScienceDirect

- University library databases

- $\quad$ Academic OneFile (Gale)

- CINAHL (EBSCO)

- Education Full Text (EBSCO)

- $\quad$ ERIC (EBSCO)

- Health Source: Nursing/Academic (EBSCO)

- JSTOR

- PubMed

- ScienceDirect (Elsevier)

- $\quad$ SPORTDiscus (EBSCO)

- Review of reference lists

\section{Inclusion and Exclusion Criteria (Include Search Limits)}

\section{Inclusion Criteria}

- Studies investigated participants with an ACL tear.

- Studies compared clinical diagnostic tests to MRI in diagnosis of ACL tears.

- Studies used arthroscopy as diagnostic gold standard.

- Level 3 evidence or higher.

- Limited to English language.

- Limited to humans.

- Limited to last 5 years (2013-2017).

\section{Exclusion Criteria}

- Studies including participants with acute injuries.

- Studies including participants with inconclusive MRI and clinical diagnostic test diagnoses.

- Studies including participants who were treated nonoperatively.

- Studies including participants with history of previous knee injury.

- Studies including participants with history of prior knee surgery.

\section{Results of Search}

Three relevant studies ${ }^{4-6}$ were identified and are listed as shown in Table 1.

\section{Best Evidence}

The studies ${ }^{4-6}$ in Table 2 were identified as the best evidence and selected for inclusion in this literature review. These studies were selected because they investigated the following:

- Participants with ACL tears
Table 1 Summary of Study Designs of Articles Retrieved

\begin{tabular}{|c|c|c|c|}
\hline $\begin{array}{l}\text { Level of } \\
\text { evidence }\end{array}$ & $\begin{array}{l}\text { Study design/methodology } \\
\text { of articles retrieved }\end{array}$ & $\begin{array}{l}\text { Number } \\
\text { located }\end{array}$ & Reference \\
\hline 2 & $\begin{array}{l}\text { Cross-sectional, descriptive } \\
\text { analytical study }\end{array}$ & 1 & $\begin{array}{l}\text { Navali } \\
\text { et } \mathrm{al}^{5}\end{array}$ \\
\hline \multirow[t]{2}{*}{3} & Local nonrandom sample & 2 & $\begin{array}{l}\text { Kostov } \\
\text { et } \mathrm{al}^{6}\end{array}$ \\
\hline & & & $\begin{array}{l}\text { Siddiqui } \\
\text { et } \mathrm{al}^{4}\end{array}$ \\
\hline
\end{tabular}

- Cross-sectional design comparing diagnostic accuracy of clinical diagnostic tests to MRI in the diagnosis of ACL tears

- The use of arthroscopy as the gold standard

\section{Implications for Practice, Education, and Future Research}

Knee injuries, specifically ACL tears, are increasing every year. ${ }^{2}$ Clinical examination of knee injuries is indicated immediately following injury. ${ }^{6}$ The clinical exam will include observation, palpation, clinical diagnostic tests, and a neurological exam. ${ }^{7}$ Positive clinical diagnostic tests should prompt the examiner to refer the patient for further diagnostic testing. Three methods of ACL injury diagnosis utilized are clinical diagnostic tests, MRI, and diagnostic arthroscopy. ${ }^{4-6}$ The 3 commonly used diagnostic tests are Lachman test, anterior drawer test, and pivot shift test. ${ }^{1,4-6}$ The Lachman test is conducted with the patient lying supine. The knee is passively flexed to 20 to 25 degrees of flexion. One of the examiner's hands is placed just superior to the femoral condyles, and the other hand grasps the patient's tibia about the tibial tuberosity. The examiner applies posterior pressure to the femur, while simultaneously attempting to translate the tibia anteriorly. When compared bilaterally, a positive Lachman test is indicated by increased anterior tibial translation or absence of a firm endpoint. ${ }^{7}$ The anterior drawer test is performed with the patient supine, hip flexed to $45^{\circ}$ and knee flexed to $90^{\circ}$. The examiner sits on the examination table near the patient's foot, placing both hands around the proximal lower leg, just inferior to the joint line of the knee. The examiner's thumbs are placed on the patient's medial and lateral joint lines on both sides of the patellar tendon. The examiner attempts to draw the patient's tibia anteriorly. When compared bilaterally, a positive anterior drawer test is indicated by increased anterior tibial translation or absence of a firm endpoint. ${ }^{8}$ When conducting the pivot shift test, the patient is supine, and the hip is passively flexed to $30^{\circ}$. The examiner stands lateral to the patient, grasps the patient's ankle, and internally rotates the tibia $20^{\circ}$. The examiner allows the knee to fully extend and places the opposite hand on the lateral aspect of the lower leg at the superior tibifibular joint. The examiner maintains tibia internal rotation, then flexes the knee and applies a valgus force to the knee. Between 30 and 40 degrees of flexion, the patient's tibia reduces on the femur, indicating a positive test. ${ }^{8}$ According to van Eck et $a{ }^{1}{ }^{1}$ when diagnosing an acute, complete ACL tear, the Lachman test has the highest sensitivity. The authors also concluded that specificity was comparable between the Lachman test, anterior drawer test, and pivot shift test. ${ }^{1}$ These findings by van Eck et $\mathrm{al}^{1}{ }^{1}$ support the 


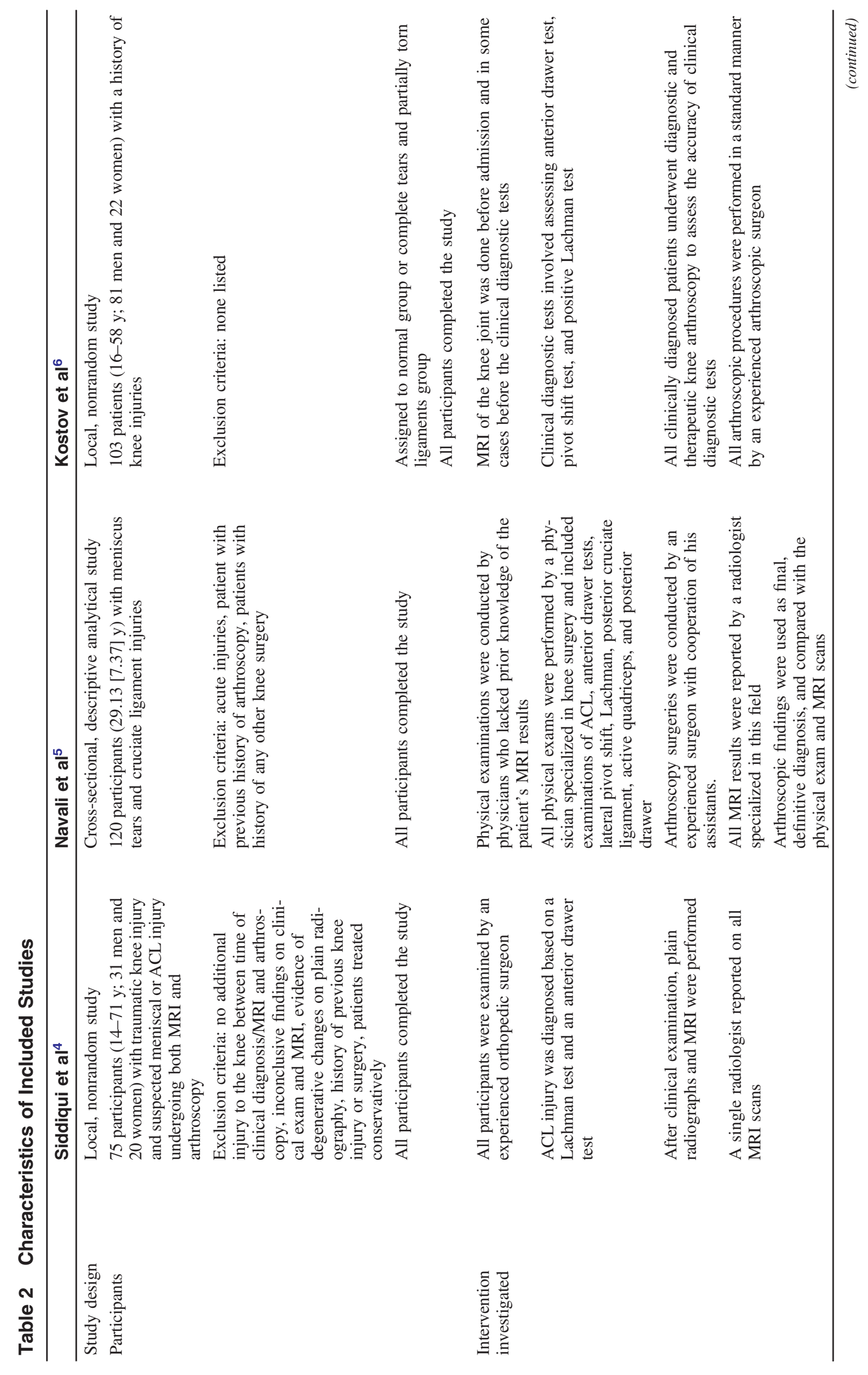




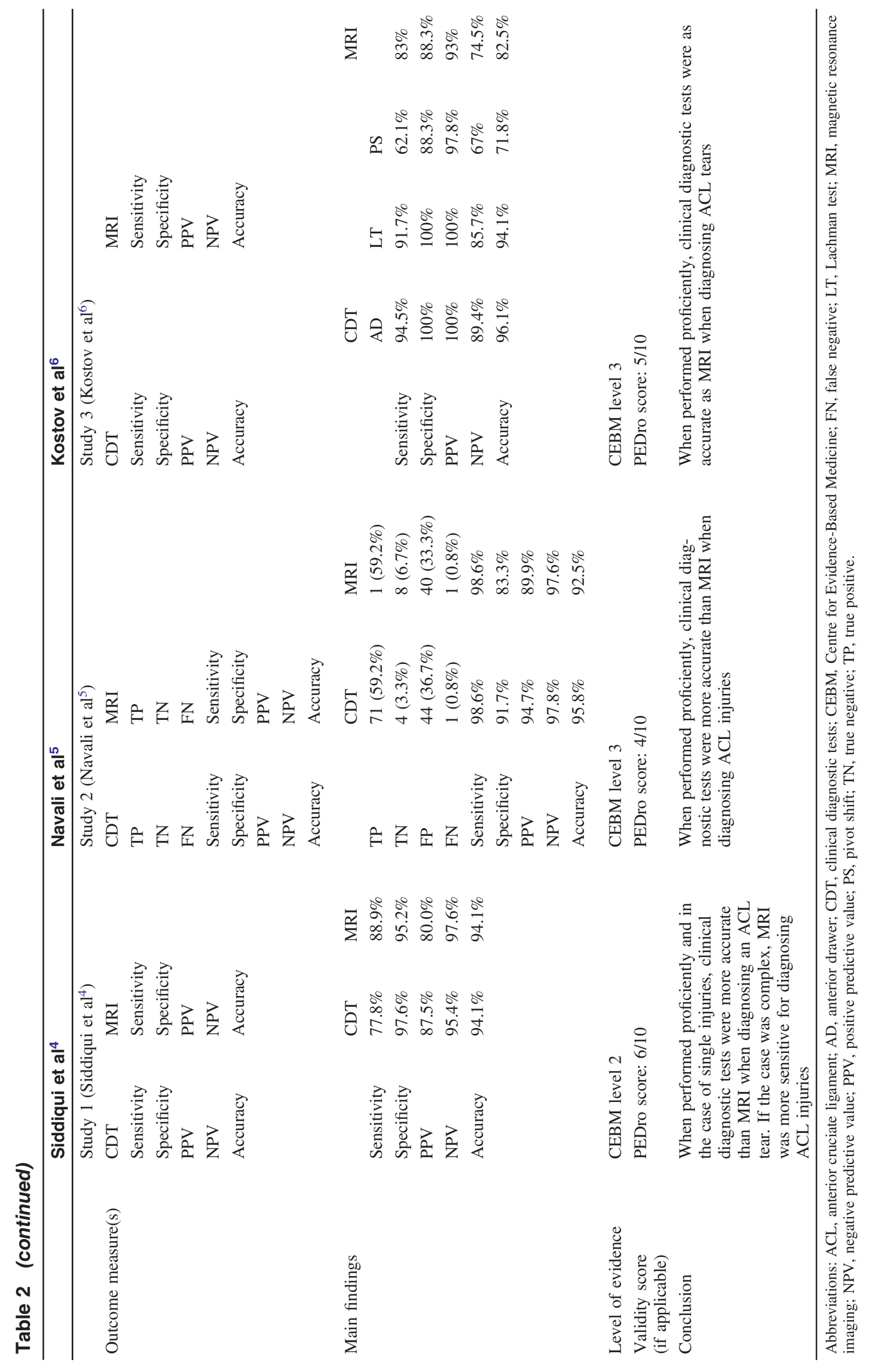


clinical diagnostic test findings of the articles reviewed in this critically appraised topic. ${ }^{4-6}$

When comparing the diagnostic accuracy of clinical diagnostic tests and MRI in the diagnosis of ACL tears, Kostov et $\mathrm{al}^{6}$ conducted a prospective study to compare clinical diagnostic tests with MRI in the diagnosis of ACL tears. Kostov et $\mathrm{al}^{6}$ found that clinical diagnostic tests were superior to the MRI in diagnosing an ACL tear in all measured categories: sensitivity (94.3\% vs $83 \%$ ), specificity ( $110 \%$ vs $88.3 \%)$, PPV (100\% vs $93 \%)$, NPV ( $89.4 \%$ vs $74.5 \%$ ), and accuracy (96.1\% vs $82.5 \%$ ). Additionally, Navali et $\mathrm{al}^{5}$ determined that, if conducted proficiently, the clinical diagnostic tests were equal to MRI when measuring sensitivity (98.6\%), but scored higher in specificity (91.7\% vs $83.3 \%)$, PPV (94.7\% vs $89.9 \%$ ), NPV (97.8\% vs $97.6 \%$ ), and diagnostic accuracy $(95.8 \%$ vs $92.5 \%)$. Siddiqui et $\mathrm{al}^{4}$ determined that clinical diagnostic tests scored higher than MRI on specificity (97.6\% vs $95.2 \%)$ and PPV (87.5\% vs $80.0 \%$ ), were equal when measuring accuracy $(94.1 \%)$, and scored lower when measuring sensitivity $(77.8 \%$ vs $88.9 \%)$ and NPV (95.4\% vs $97.6 \%)$.

The ability to diagnose an ACL tear using clinical diagnostic tests could have major implications for the patient. Diagnosing an ACL tear accurately using clinical diagnostic tests could disqualify the patient from further physical or work-related activity and prevent increased trauma to the knee. ${ }^{9,10}$ Additionally, if an ACL tear is promptly and accurately diagnosed with clinical diagnostic tests, preoperative rehabilitation can be initiated immediately, improving patient functional outcomes and return to sport rates. ${ }^{11}$ Moreover, an MRI can be a costly diagnostic test, and the sensitivity is variable and based on the ability of the examiner. ${ }^{3}$ According to Kostov et al, ${ }^{6}$ if an ACL tear is diagnosed clinically, an MRI is an unnecessary and expensive test. A clinically diagnosed ACL tear may not warrant an MRI, due to lack of significance, ${ }^{6}$ and if the clinical diagnostic tests are accurate, a negative clinical exam could negate the need for the MRI. Accurately diagnosing or ruling out an ACL tear with clinical diagnostic tests could save the patient, patient's family, employer, or school money by avoiding an MRI. Early diagnosis and prevention of additional knee trauma, ${ }^{9}$ along with preoperative rehabilitation, ${ }^{11}$ and cost savings may lead to improved patient outcomes.

Future research is necessary to determine if clinical diagnostic tests are more diagnostically accurate than MRI in the diagnosis of ACL tears. More studies will need to be conducted to compare specificity, sensitivity, PPV, NPV, and accuracy of clinical diagnostic tests and MRI, as well as clinical diagnostic tests proficiency of the examiner.

\section{References}

1. van Eck CF, van den Bekerom MPJ, Fu FH, Poolman RW, Kerkhoff GMMJ. Methods to diagnose acute anterior cruciate ligament rupture: a meta-analysis of physical examinations with and without anaesthesai. Knee Surg Sports Traumatol Arthrosc. 2013;21:1895-1903. PubMed: 23085822 doi:10.1007/s00167-0122250-9

2. Sanders TL, Kremers HM, Bryan AJ, et al. Incidence of anterior cruciate ligament tears and reconstruction: a 21-year populationbased study. Am J Sport Med. 2016;44(6):1502-1507. doi:10. 1177/0363546516629944

3. Vaz CE, Camango OP, Santana JP, et al. Accuracy of magnetic resonance in identifying traumatic intraarticular knee lesions. Clinics. 2005;60(6):445-450. doi:10.1590/S1807-59322005000600003

4. Siddiqui MA, Ahmad I, Bin Sabir A, Ullah E, Rizvi SAA, Rizvi SWA. Clinical examination vs MRI: evaluation of diagnostic accuracy in detecting ACL and meniscal injuries in comparison to arthroscopy. Pol Orthop Traumatol. 2013;78:59-63. PubMed: 23416722

5. Navali AM, Bazavar M, Mohseni MA, Safari B, Tabrizi A. Arthroscopic evaluation of the accuracy of clinical examination versus MRI in diagnosing meniscus tears and cruciate ligament ruptures. Arch Iran Med. 2013;16(4):229-232. PubMed: 23496367

6. Kostov H, Arsovski O, Kostova E, Nikilov V. Diagnostic assessment in anterior cruciate ligament (ACL) tears. Prilozi. 2014;35(1):209218. PubMed: 24798607

7. Starkey C, Ryan J. Evaluation of Orthopedic and Athletic Injuries. 2nd ed. Philadelphia, PA: F.A. Davis Company; 2002.

8. Dejour D, Ntagiopoulos PG, Sagin PR, Paniset J-C. The diagnostic value of clinical tests, magnetic resonance imaging, and instrumented laxity in the differentiation of complete versus partial anterior cruciate ligament tears. Arthroscopy. 2013;29(3):491-499. PubMed: 23343713 doi:10.1016/j.arthro.2012.10.013

9. Lintner DM, Kamaric E, Moseley JB, Noble PC. Partial tears of the anterior cruciate ligament. Are they clinically detectable? Sports Med. 1995;23(1):111-118. doi:10.1177/036354659502300119

10. Risbert MA, Oiestad BE, Gunderson R, et al. Changes in knee osteoarthritis, symptoms, and function after anterior cruciate ligament reconstruction. Am J Sport Med. 2016;44(5):1215-1224. doi:10. 1177/0363546515626539

11. Lelli A, Di Turi RP, Spenciner DB, Dòmini M. The "Lever Sign": a new clinical test for the diagnosis of anterior cruciate ligament rupture. Knee Surg Sports Traumatol Arthrosc. 2016;24:27942797. PubMed: 25536951 doi:10.1007/s00167-014-3490-7 